\title{
MRI data-driven algorithm for the diagnosis of behavioral variant
}

\section{frontotemporal dementia}

Ana L. Manera ${ }^{1}$; Mahsa Dadar ${ }^{1}$; John van Swieten ${ }^{3}$, Barbara Borroni ${ }^{4}$, Raquel Sanchez-Valle ${ }^{5}$, Fermin Moreno ${ }^{6}$, Robert LaForce $\mathrm{Jr}^{7}$, Caroline Graff ${ }^{8}$, Matthis Synofzik ${ }^{9}$, Daniela

Galimberti $^{10,11}$, James Rowe ${ }^{12}$, Mario Masellis ${ }^{13}$, Maria Carmela Tartaglia ${ }^{14}$, Elizabeth Finger ${ }^{15}$, Rik Vandenberghe ${ }^{16}$, Alexandre de Mendonça ${ }^{17}$, Fabrizio Tagliavini ${ }^{18}$, Isabel Santana ${ }^{19}$, Chris

Butler $^{20}$, Alex Gerhard ${ }^{21}$, Adrian Danek ${ }^{22}$, Johannes Levin ${ }^{22}$, Markus Otto ${ }^{23}$, Giovanni

Frisoni $^{24,25}$, Roberta Ghidoni ${ }^{26}$, Sandro Sorbi ${ }^{27,28}$, Jonathan D Rohrer ${ }^{29}$; Simon Ducharme ${ }^{1,2^{*}}$ and

D. Louis Collins ${ }^{1 *}$

Frontotemporal Lobar Degeneration Neuroimaging Initiative (FTLDNI) ${ }^{\dagger}$

GENetic Frontotemporal dementia Initiative (GENFI)

*These authors contributed equally to this work (shared senior authorship).

\section{Author's Affiliations:}

${ }^{1}$ McConnell Brain Imaging Centre, Montreal Neurological Institute, McGill University, Montreal, Quebec (QC), Canada. 3801, University, Montreal, Quebec, H3A 2 B4.

${ }^{2}$ Douglas Mental Health University Institute, Department of Psychiatry, 6875 Boulevard LaSalle, Verdun, QC H4H 1R3

${ }^{3}$ Department of Neurology, Erasmus Medical Center, Rotterdam, The Netherlands

${ }^{4}$ Centre for Neurodegenerative Disorders, Department of Clinical and Experimental Sciences, University of Brescia, Brescia, Italy

5 Alzheimer's disease and Other Cognitive Disorders Unit, Neurology Service, Hospital Clínic, Institut d'Investigacións Biomèdiques August Pi I Sunyer, University of Barcelona, Barcelona, Spain

6 Cognitive Disorders Unit, Department of Neurology, Donostia University Hospital, San Sebastian, Gipuzkoa, Spain 
${ }^{7}$ Clinique Interdisciplinaire de Mémoire, Département des Sciences Neurologiques, CHU de Québec, and Faculté de Médecine, Université Laval, Quebec, Canada

8 Department of Geriatric Medicine, Karolinska University Hospital-Huddinge, Stockholm, Sweden

${ }^{9}$ Department of Neurodegenerative Diseases, Hertie-Institute for Clinical Brain Research and Center of Neurology, University of Tübingen, Tübingen, Germany

${ }^{10}$ Fondazione IRCCS Ca' Granda Ospedale Maggiore Policlinico, Neurodegenerative Diseases Unit, Milan, Italy

${ }^{11}$ LANE - Laboratory of Alzheimer's Neuroimaging and Epidemiology, IRCCS Istituto Centro San Giovanni di Dio Fatebenefratelli, Brescia, Italy

${ }^{12}$ Department of Clinical Neurosciences, University of Cambridge, Cambridge, UK

13 Sunnybrook Health Sciences Centre, Sunnybrook Research Institute, University of Toronto, Toronto, Canada

14 Toronto Western Hospital, Tanz Centre for Research in Neurodegenerative Disease, Toronto, Ontario, Canada

${ }^{15}$ Department of Clinical Neurological Sciences, University of Western Ontario, London, ON, Canada

${ }^{16}$ Laboratory for Cognitive Neurology, Department of Neurosciences, KU Leuven, Leuven, Belgium

${ }^{17}$ Faculty of Medicine, University of Lisbon, Lisbon, Portugal

${ }^{18}$ Fondazione Istituto di Ricovero e Cura a Carattere Scientifico Istituto Neurologico Carlo Besta, Milan, Italy

${ }^{19}$ Neurology Department, Centro Hospitalar e Universitário de Coimbra, Coimbra, Portugal

${ }^{20}$ Department of Clinical Neurology, University of Oxford, Oxford, UK

21 Institute of Brain, Behaviour and Mental Health, The University of Manchester, Withington, Manchester, UK

22 Neurologische Klinik und Poliklinik, Ludwig-Maximilians-Universität, Munich, German Center for Neurodegenerative Diseases (DZNE), Munich, Germany

${ }^{23}$ Department of Neurology, University Hospital Ulm, Ulm, Germany

${ }^{24}$ LANE - Laboratory of Alzheimer's Neuroimaging and Epidemiology, IRCCS Istituto Centro San Giovanni di Dio Fatebenefratelli, Brescia, Italy 
${ }^{25}$ Memory Clinic and LANVIE-Laboratory of Neuroimaging of Aging, University Hospitals and University of Geneva, Geneva, Switzerland

${ }^{26}$ Molecular Markers Laboratory, IRCCS Istituto Centro San Giovanni di Dio Fatebenefratelli, Brescia, Italy

27 Department of Neuroscience, Psychology, Drug Research and Child Health, University of Florence, Florence, Italy

${ }^{28}$ Department of Neuroscience, Psychology, Drug Research, and Child Health, University of Florence, Florence, Italy

29 Department of Neurodegenerative Disease, Dementia Research Centre, UCL Institute of Neurology, Queen Square, London, UK

$\dagger$ Data used in preparation of this article were obtained from the Frontotemporal Lobar Degeneration Neuroimaging Initiative (FTLDNI) database (http://4rtni-ftldni.ini.usc.edu/ ). The investigators at NIFD/FTLDNI contributed to the design and implementation of FTLDNI and/or provided data but did not participate in analysis or writing of this report (unless otherwise listed). The FTLDNI investigators included the following individuals:

Howard Rosen; University of California, San Francisco (PI)

Bradford C. Dickerson; Harvard Medical School and Massachusetts General Hospital

Kimoko Domoto-Reilly; University of Washington School of Medicine

David Knopman; Mayo Clinic, Rochester

Bradley F. Boeve; Mayo Clinic Rochester

Adam L. Boxer; University of California, San Francisco

John Kornak; University of California, San Francisco

Bruce L. Miller; University of California, San Francisco

William W. Seeley; University of California, San Francisco

Maria-Luisa Gorno-Tempini; University of California, San Francisco

Scott McGinnis; University of California, San Francisco

Maria Luisa Mandelli; University of California, San Francisco

$\$$ List of other GENFI Consortium Members in Appendix

\section{Corresponding Author Information:}

Ana L. Manera, Montreal Neurological Institute, 3801 University Street, Room WB320, Montréal, QC, H3A 2B4

Email: ana.manera@mcgill.ca; ana.manera@mail.mcgill.ca

\section{Word Count: 3741}




\begin{abstract}
Introduction: Structural brain imaging is paramount for the diagnosis of behavioral variant of frontotemporal dementia (bvFTD), but it has low sensitivity leading to erroneous or late diagnosis. Methods: A total of 515 subjects from two different bvFTD cohorts (training and independent validation cohorts) were used to perform voxel-wise morphometric analysis to identify regions with significant differences between bvFTD and controls. A random forest classifier was used to individually predict bvFTD from deformation-based morphometry differences in isolation and together with semantic fluency. Ten-fold cross validation was used to assess the performance of the classifier within the training cohort. A second held-out cohort of genetically confirmed bvFTD cases was used for additional validation.

Results: Average ten-fold cross-validation accuracy was $89 \%$ (82\% sensitivity, 93\% specificity) using only MRI and 94\% (89\% sensitivity, 98\% specificity) with the addition of semantic fluency. In the separate validation cohort of definite bvFTD, accuracy was $88 \%$ (81\% sensitivity, $92 \%$ specificity) with MRI and 91\% (79\% sensitivity, 96\% specificity) with added semantic fluency scores.

Conclusion: Our results show that structural MRI and semantic fluency can accurately predict bvFTD at the individual subject level within a completely independent validation cohort coming from a different and independent database.
\end{abstract}

\title{
Keywords
}

Frontotemporal dementia -Magnetic resonance -Deformation-based morphometry Classification- Machine learning 


\section{Abbreviations}

FTD: frontotemporal dementia

$G R N$ : progranulin

MAPT: microtubule-associated protein tau

C9orf72 : chromosome 9 open reading frame 72

bvFTD: behavioural variant of frontotemporal dementia

CNCs: cognitively normal controls

DBM: deformation-based morphometry

FTLDNI: frontotemporal lobar degeneration neuroimaging initiative

FTLD: frontotemporal lobar degeneration

T1w: T1 weighted

GENFI: Genetic frontotemporal dementia initiative

MMSE: Mini mental state examination

MoCA: Montreal cognitive assessment

FTLD-CDR: Frontotemporal lobar degeneration Clinical Dementia Rating score

CGI: Clinical global impression

FRS: Frontotemporal dementia rating scale

FDR: False Discovery Rate

PCA: Principal component analysis

PCs: Principal components

SF: Semantic fluency

ROC: Receiver operating characteristic curves

AUC: Area under the curve

LR+: positive likelihood ratio

LR-: negative likelihood ratio 


\section{INTRODUCTION}

The heterogeneity of frontotemporal dementia (FTD) is a hallmark of the disease with significant variations in heritability, pathology and clinical presentations. ${ }^{1}$ First, although most cases of FTD are sporadic, $10-30 \%$ are caused by an autosomal dominant mutation (most commonly progranulin -GRN-, microtubule-associated protein Tau -MAPT- and chromosome 9 open reading frame 72 C9orf72-). ${ }^{23}$ Second, in terms of the underlying pathology, there are three main groups according to the major protein involved, all of which are characterized by selective degeneration of the frontal and temporal lobes: Tau, transactive response DNA-binding protein of $43 \mathrm{kDa}-\mathrm{TDP}-43-$, and the tumor associated protein fused in sarcoma -FUS-. ${ }^{4}$ In the absence of molecular biomarkers, and when combined with the syndromic overlap with other neurodegenerative disorders and psychiatric disorders, a confirmed behavioral variant frontotemporal dementia (bvFTD) diagnosis is often difficult to achieve and heavily relies on brain imaging. ${ }^{6}$

While the presence of fronto-temporal atrophy on MRI increases the level of diagnostic confidence and has high specificity, it lacks sensitivity particularly in the initial stages of the disease, leading to erroneous or late diagnosis. ${ }^{78}$ It is therefore necessary to extract MRI features that have better discriminatory power to aid in diagnosis. Recently, machine learning techniques have been applied to distinguish between bvFTD and Cognitively Normal Subjects (CNCs), Alzheimer Disease or other psychiatric and neurologic disorders on an individual level using MRI-based features.9-17 These studies vary greatly on their population and methodology. In general, they achieved moderate to high accuracy distinguishing bvFTD. However, sample sizes were small, training and test cohorts did not come from the independent datasets and, therefore, the clinical applicability remains to be determined. ${ }^{18}$ Further, it is uncertain if these classifiers would work in a clinical 
population including genetic bvFTD cases in which the MRI patterns of atrophy share similarities with sporadic cases, but also have distinctive features for each mutation. ${ }^{19-21}$

In the present study, we developed a Random Forest classifier ${ }^{22}$ using features derived from Deformation Based Morphometry (DBM) maps to identify bvFTD subjects from CNCs. To ensure the generalizability of the results, the machine learning model was trained on a mainly sporadic cohort and tested in a held-out population of genetic bvFTD, therefore relying on one of the gold standards for diagnosis (i.e., definite bvFTD). ${ }^{7}$

\section{MATERIALS AND METHODS}

\section{Participants}

A total of 515 subjects were examined in this study. The first cohort was used only for training (the 'training cohort'). The training cohort included bvFTD patients and CNCs from the Frontotemporal Lobar Degeneration Neuroimaging Initiative (FTLDNI) database who had T1weighted (T1w) MRI scans matching with each clinical visit. The inclusion criteria for bvFTD patients was a diagnosis of possible or probable bvFTD according to the FTD consortium criteria ${ }^{7}$, resulting in 70 patients with bvFTD and 123 CNCs in our study.

The primary goals of the FTLDNI, funded through the National Institute of Aging, are to identify neuroimaging modalities and methods of analysis for tracking frontotemporal lobar degeneration (FTLD) and to assess the value of imaging versus other biomarkers in diagnostic roles. For up-todate information on participation and protocol, please visit: http://4rtni-ftldni.ini.usc.edu/ . 
The second cohort was completely independent from the first and used only for validation (the 'validation cohort') of the model created with the training cohort. The validation cohort included bvFTD patients and CNCs from the third data freeze (12/2017) of the Genetic Frontotemporal Dementia Initiative 2 (GENFI2- http://genfi.org.uk/), ${ }^{23}$ which includes 23 centres in the UK, Europe and Canada. GENFI2 participants included known symptomatic carriers of a pathogenic mutation in $C 9 \operatorname{orf72,} G R N$ or $M A P T$ and their first-degree relatives who are at risk of carrying a mutation, but who did not show any symptoms (i.e., presymptomatic). Non-carriers were firstdegree relatives of symptomatic carriers who did not carry the mutation. The inclusion and exclusion criteria are described in detail elsewhere. ${ }^{23}$ Since the aim of the present study was to differentiate bvFTD patients from CNCs, presymptomatic carriers and symptomatic carriers whose clinical diagnosis was other than bvFTD were excluded. The CNC group consists of subjects who are first degree relatives of patients with FTD genetic mutations, but who are asymptomatic and were tested negatively for the mutation that is present in their family. This validation cohort contained 75 patients with bvFTD and 247 CNCs and was never used for feature selection, parameter identification or model tuning during the training phase.

\section{Clinical assessment}

All subjects were regularly assessed clinically yearly/every six-months by site investigators. Neuropsychological assessment included Mini Mental State Examination (MMSE), Montreal Cognitive Assessment (MoCA), FTLD clinical dementia rating (FTLD-CDR), Clinical Global Impression (CGI), verbal fluency, Frontotemporal dementia rating scale (FRS) amongst other cognitive and functional scores ${ }^{24-28}$. 


\section{Image acquisition and preprocessing}

For the FTLDNI training cohort, 3.0T MRIs were acquired at three sites (T1w MPRAGE, TR=2 ms, TE=3 ms, IT=900 ms, flip angle $9^{\circ}$, matrix 256x240, slice thickness $1 \mathrm{~mm}$, voxel size $1 \mathrm{~mm}^{3}$ ).

For the GENFI2 validation cohort, participants underwent volumetric T1w MPRAGE MRI at multiple centers, according to the GENFI imaging protocol using either Siemens Trio 3T, SiemensSkyra3T, Siemens1.5T, Phillips3T, General Electric (GE) 1.5T or GE 3T scanners. Scan protocols were designed at the outset of the study to ensure adequate matching between the scanners and image quality control.

The T1w scans of the subjects were pre-processed through our longitudinal pipeline ${ }^{29}$ that included image denoising, ${ }^{30}$ intensity non-uniformity correction $^{31}$, and image intensity normalization into range $(0-100)$ using histogram matching. Each native T1w volume from each timepoint was linearly registered first to the subject-specific template which was then registered to the ICBM152 template. ${ }^{32}$ All images were then non-linearly registered to the ICBM152 template using ANTs diffeomorphic registration pipeline. ${ }^{33}$ The images were visually assessed by two experienced raters to exclude cases with significant imaging artifacts (e.g. motion, incomplete field of view) or inaccurate linear/nonlinear registrations. This visual assessment was performed blind to diagnosis. Out of 1724 scans, only 43 (2.5\%, 36 scans in GENFI2, and 7 in FTLDNI) did not pass this visual quality control. For the purpose of this study, scans from subjects other than bvFTD or CNCs, or those that did not have a matching clinical visit were excluded from this analysis. This resulted in a total of 515 subjects that were included in this study. 


\section{Deformation based morphometry}

DBM ${ }^{34} 35$ analysis was performed using Montreal Neurological Institute (MNI) MINC tools. ${ }^{29}$ The local deformation obtained from the non-linear transformations was used as a measure of tissue expansion or atrophy by computing the determinant of the Jacobian at each voxel. Local contractions can be interpreted as shrinkage (e.g., tissue atrophy) and local expansions are often related to ventricular or sulci enlargement. DBM was used to assess both voxel-wise and atlasbased cross-sectional group related volumetric differences.

\section{Classification bvFTD versus CNCs}

To obtain a region of interest map reflecting the patterns of difference between bvFTD and CNCs, a voxel-wise mixed effects model analysis was performed only within the training dataset. The mixed effects model included age as a continuous fixed variable and diagnosis and sex as fixed categorical variables. Subject was included as a categorical random variable. The variable of interest was diagnosis, reflecting the brain regions that were significantly different between bvFTD and CNCs, controlling for age and sex. The results were corrected for multiple comparisons using the False Discovery Rate (FDR) with a $\mathrm{P}$ value $<0.05$ threshold. Figure 1 shows the resulting map reflecting areas of significant difference between bvFTD and CNCs. ${ }^{36}$ 

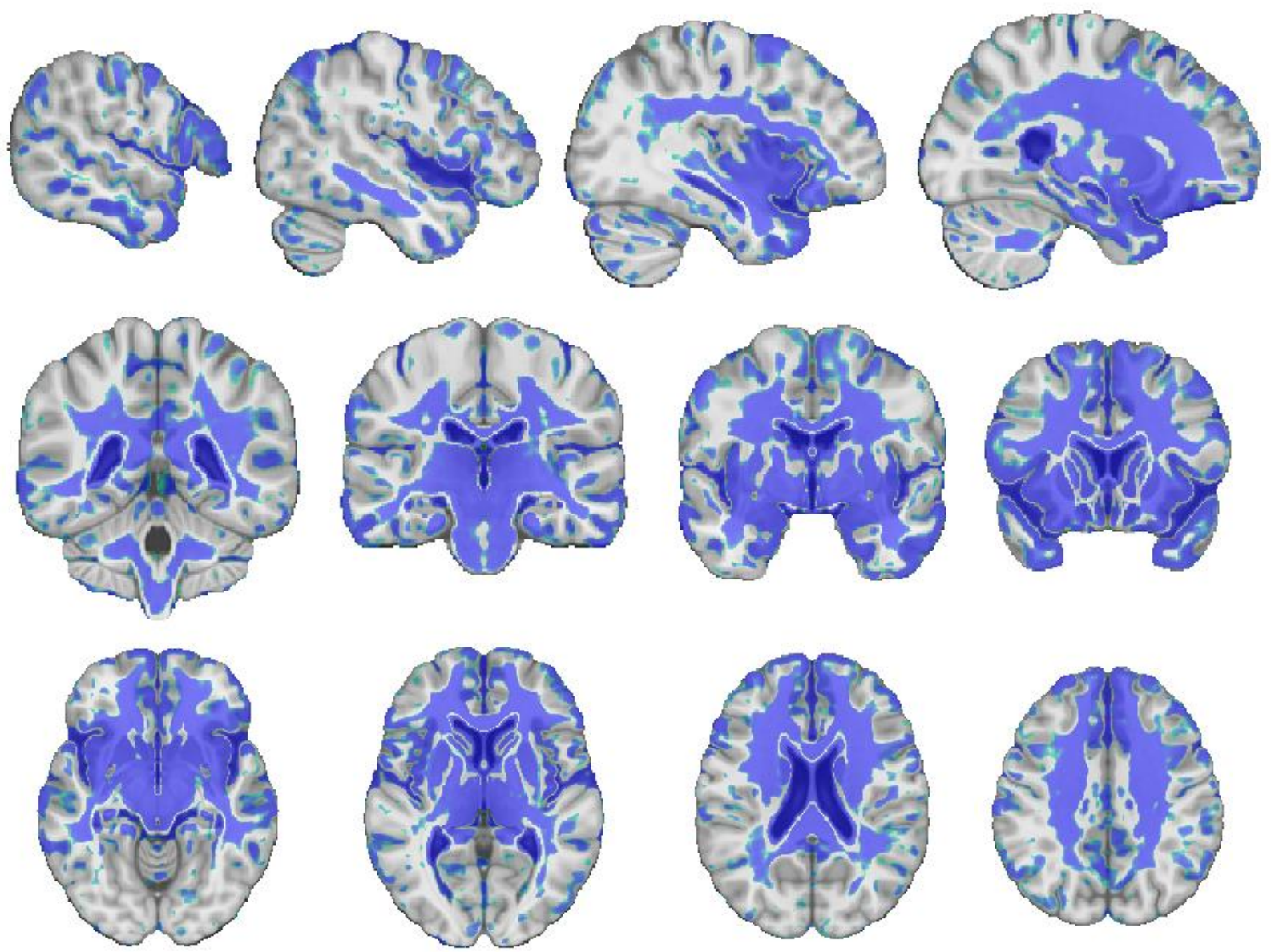

Figure 1. Region of interest map reflecting the significant patterns of difference between bvFTD and CNCs, obtained based on the training data.

A principal component analysis (PCA) was then performed on the DBM voxels within this region of interest. To avoid any leakage (i.e., double dipping), only the baseline information from the training data was used for this PCA. Two sets of features were then used to train a random forests classifier ${ }^{22}$ with 500 trees: 1) the first five principal components (PCs, selected based on scree plots of the obtained eigenvalues) as well as age and sex, and 2) the first five PCs, age, sex, and a neuropsychological score. The Semantic Fluency score (SF) was used as the cognitive score feature since it is a reliable simple bedside test associated with executive and language deficits in 
bvFTD ${ }^{37}$ and was available for most of the subjects in both training and validation datasets. Executive deficits are considered a core characteristic of FTD, though in themselves, are insufficient to establish a diagnosis and thus not used here. ${ }^{38}{ }^{39}$ Classifications were run using $\mathrm{DBM}$ in isolation and DBM $+\mathrm{SF}$. Ten-fold cross validation was used to assess the performance of the classifier within the training data. To obtain a single ROI and consistent features across folds, the voxel-wise group comparison and PCA were performed outside of the cross-validation loop on the training dataset, since we use an independent dataset for validation. However, to demonstrate that this choice does not lead to leakage in the training set experiments, we also repeated the cross validation for the training set with the mixed effects model and PCA analyses performed inside the cross-validation loop and obtained similar results (see the supplementary materials for details).

To perform classification on the held-out GENFI2 validation dataset, the coefficients calculated based on the PCA on the training dataset were used to calculate the first five PCs features for the subjects from the validation dataset. Using the random forest classifier trained on FTLDNI, we then classified all the subjects from the validation dataset as either bvFTD or CNCs (based on their baseline information). A probability score was also obtained from the random forest classifier, indicating the likelihood of each observation belonging to the bvFTD class. The mixed effects modelling, PCA, and random forest classification were carried out using MATLAB (version R2017b).

\section{Statistical analyses}


All statistical analyses were conducted using MATLAB (version R2017b). Two-sample t-Tests were conducted to examine demographic and clinical variables at baseline. Categorical variables were analysed using chi-square analyses. Results are expressed as mean \pm standard deviation and median [interquartile range] as appropriate. Receiver Operator Characteristics (ROC) analysis was used to define sensitivity and specificity at different cut-points within the validation cohort. The optimal cut-point was estimated by the use of Youden index ( $\mathrm{J}=$ Sensitivity+Specificity-1). Positive and negative likelihood ratios were also estimated for different cut points.

\section{RESULTS}

\section{Demographics}

Table 1 shows the demographic and cognitive testing performances in bvFTD and CNCs. There was no difference in age between bvFTD patients and CNCs (62 \pm 6 and $63 \pm 6$ years respectively, $\mathrm{p}=0.36)$, but there was a higher proportion of males in bvFTD patients than CNCs (67\% vs $43 \%$, $\mathrm{p}=0.001)$. As expected, bvFTD subjects showed greater cognitive and functional impairment: significant differences were found between the two cohorts in MMSE, FTLD-CDR, MoCA, letter fluency Z-score and semantic fluency Z-score (all p < 0.001).

Demographic differences and cognitive testing performances between patients and controls for the GENFI cohort are also shown in Table 1. Considering the CNCs from this dataset comes from non-carrier members of families at risk of genetic mutation related to FTD, they were, as expected, significantly younger than bvFTD subjects. The mean age was $48 \pm 14$ years for CNCs and $64 \pm 8$ years for bvFTD $(\mathrm{p}<.001)$. The median disease duration (age at visit - age of symptom onset) for the bvFTD group was 5.1[3.5-8.2] and the estimated years to onset (age at baseline - average age 
of disease onset in the family) 5.2 \pm 5.7 years. Compared to non-carriers, bvFTD subjects showed greater cognitive and functional impairment. Significant differences were found between the two cohorts in MMSE, FTLD-CDR, MoCA, FRS, letter fluency Z-score and semantic fluency Z-score $(\mathrm{p}<.001)$. Regarding the mutated gene, half of the bvFTD subjects carried a C9orf72 mutation, while $22.7 \%$ and $25.3 \%$ belonged to the $M A P T$ and $G R N$ groups respectively. 
Table 1. Demographic and clinical characteristics in bvFTD and healthy controls

\begin{tabular}{|c|c|c|c|c|c|c|c|}
\hline & \multicolumn{3}{|c|}{$\begin{array}{l}\text { Training cohort (FTLDNI) } \\
\qquad \mathrm{N}=193\end{array}$} & \multicolumn{3}{|c|}{$\begin{array}{l}\text { Validation cohort (GENFI) } \\
\qquad \mathrm{N}=322\end{array}$} \\
\hline & & \multirow{2}{*}{$\begin{array}{c}\mathrm{CNCs} \\
\mathrm{N}=123\end{array}$} & \multirow{2}{*}{$\begin{array}{l}\text { bvFTD } \\
\mathrm{N}=70\end{array}$} & \multirow[t]{2}{*}{$\mathrm{P}$ value } & \multirow{2}{*}{$\begin{array}{l}\mathrm{CNCs}^{*} \\
\mathrm{~N}=247\end{array}$} & \multirow{2}{*}{$\begin{array}{l}\text { bvFTD } \\
\mathrm{N}=75\end{array}$} & \multirow[t]{2}{*}{$\mathrm{P}$ value } \\
\hline & & & & & & & \\
\hline \multicolumn{2}{|l|}{ Age, $y$} & $63 \pm 6$ & $62 \pm 6$ & 0.36 & $48 \pm 14$ & $64 \pm 8$ & $<0.001$ \\
\hline \multicolumn{2}{|c|}{ Male sex } & $53(43 \%)$ & $47(67 \%)$ & 0.001 & $106(43 \%)$ & $41(55 \%)$ & 0.07 \\
\hline \multicolumn{2}{|c|}{ Education, y } & $17.5 \pm 1.9$ & $15.6 \pm 3.4$ & $<0.001$ & $13.9 \pm 3.5$ & $11.8 \pm 4.03$ & $<0.001$ \\
\hline \multicolumn{2}{|c|}{ Estimated years of onset, y } & - & N/A & - & - & $5.2 \pm 5.7$ & - \\
\hline \multicolumn{2}{|c|}{ Disease duration, y } & & & & & $5.1[3.5-8.2]$ & - \\
\hline \multicolumn{2}{|c|}{ MMSE score } & $29.4 \pm 0.8$ & $23.6 \pm 4.9$ & $<0.001$ & $29.4 \pm 1.1$ & $21.9 \pm 7.2$ & $<0.001$ \\
\hline \multicolumn{2}{|c|}{ FTLD-CDR Score } & $0.04 \pm 0.2$ & $6.3 \pm 3.3$ & $<0.001$ & $0.21 \pm 0.7$ & $9.7 \pm 1.4$ & $<0.001$ \\
\hline \multicolumn{2}{|c|}{ MoCA Score } & $23.6 \pm 11$ & $16.8 \pm 8.3$ & $<0.001$ & N/A & N/A & \\
\hline \multicolumn{2}{|l|}{ FRS \% } & N/A & N/A & & $88.01 \pm 28.7$ & $33.5 \pm 26.6$ & $<0.001$ \\
\hline \multicolumn{2}{|c|}{ Letter Fluency Z-score } & $0.7 \pm 0.7$ & $-0.9 \pm 0.6$ & $<0.001$ & $-0.03 \pm 1$ & $-1.3 \pm 1.4$ & $<0.001$ \\
\hline \multicolumn{2}{|c|}{ Semantic Fluency Z-score } & $0.6 \pm 0.6$ & $-1.06 \pm 0.7$ & $<0.001$ & $0.1 \pm 1$ & $-2.2 \pm 1.02$ & $<0.001$ \\
\hline Genetic & C9orf72 & - & - & & & $39(52 \%)$ & \\
\hline \multirow[t]{2}{*}{ Group } & MAPT & - & - & & & $17(22.7 \%)$ & \\
\hline & GRN & - & - & & & $19(25.3 \%)$ & \\
\hline
\end{tabular}

*CNCs in GENFI2 cohort correspond to non-carrier first degree relative of a family member with a documented genetic mutation related to FTD

Values expressed as mean \pm standard deviation, median [interquartile range]. Data available is specified for each clinical variable as N. N/A: data not available from the original databases.

FTLD: frontotemporal lobar degeneration neuroimaging initiative; GENFI: genetic frontotemporal dementia initiative; bvFTD: behavioural-variant frontotemporal dementia. CNCs: cognitively normal controls; MMSE: Mini Mental State Examination. FTLD-CDR: Frontotemporal lobar degeneration clinical dementia rating. MoCA: Montreal Cognitive Assessment. CGI: Clinical Global Impression. FRS: Frontotemporal dementia rating scale.

\section{Voxel-wise DBM group differences.}

Greater gray and white matter atrophy were found in the medial and inferior lateral portions of the frontal lobes as well as dorsolateral prefrontal cortex, insula, basal ganglia, medial and anterior 
temporal regions bilaterally and regions of brainstem and cerebellum in bvFTD. Correspondingly, volume increase was shown in the ventricles and sulci, being more evident in frontal horns and lateral sulcus. ${ }^{36}$ Supplementary material shows the high similarity (Dice similarity index (SI) of $0.93 \pm 0.01$ ) between DBM maps when mixed effects model and PCA steps are included inside each loop of the 10-fold validation compared to the DBM map in Figure S1.

\section{Random forest classification}

\section{Cross-validation results within the training cohort (FTLDNI)}

The accuracy achieved for discrimination between bvFTD and CNCs using solely morphometric MRI features (DBM) was $89 \%$, with a sensitivity of $82 \%$ and specificity of $93 \%$. When adding one cognitive score (i.e., DBM+SF) the classifier accuracy reached $94 \%$, with $89 \%$ sensitivity and 98\% specificity. When mixed effects model and PCA steps are included inside each loop of the 10-fold validation, the average accuracy is $89 \%$ using DBM features and $93 \%$ when adding semantic fluency (details in supplementary material).

\section{Classification within the validation cohort (GENFI2) using solely DBM and DBM + SF}

The model resulted in an accuracy of $88 \%$ when discriminating bvFTD patients from CNCs when applied to the independent validation cohort. Sensitivity and specificity were $81 \%$ and $92 \%$, respectively using a probability score with an optimal cut point of 0.4 as threshold. This led to a positive likelihood ratio (LR+) of 10.13 and negative likelihood ratio (LR-) of 0.21.

The inclusion of semantic fluency in the classification model resulted in an accuracy of $91 \%$, sensitivity of $79 \%$ and specificity of $96 \%$; resulting in LR+ of 19.75 and LR- of 0.22 . The ROC 
for DBM and DBM + SF classifiers are shown in Figure 2. Figure 3 shows the true positive rates for bvFTD and CNCs according the probability score for DBM (panel A) and DBM+SF (panel B). Table 2 shows the corresponding accuracy, sensitivity, specificity and likelihood ratios for the two models (DBM and DBM+SF) using different thresholds on the probability scores (e.g., for probability scores $>0.4)$.

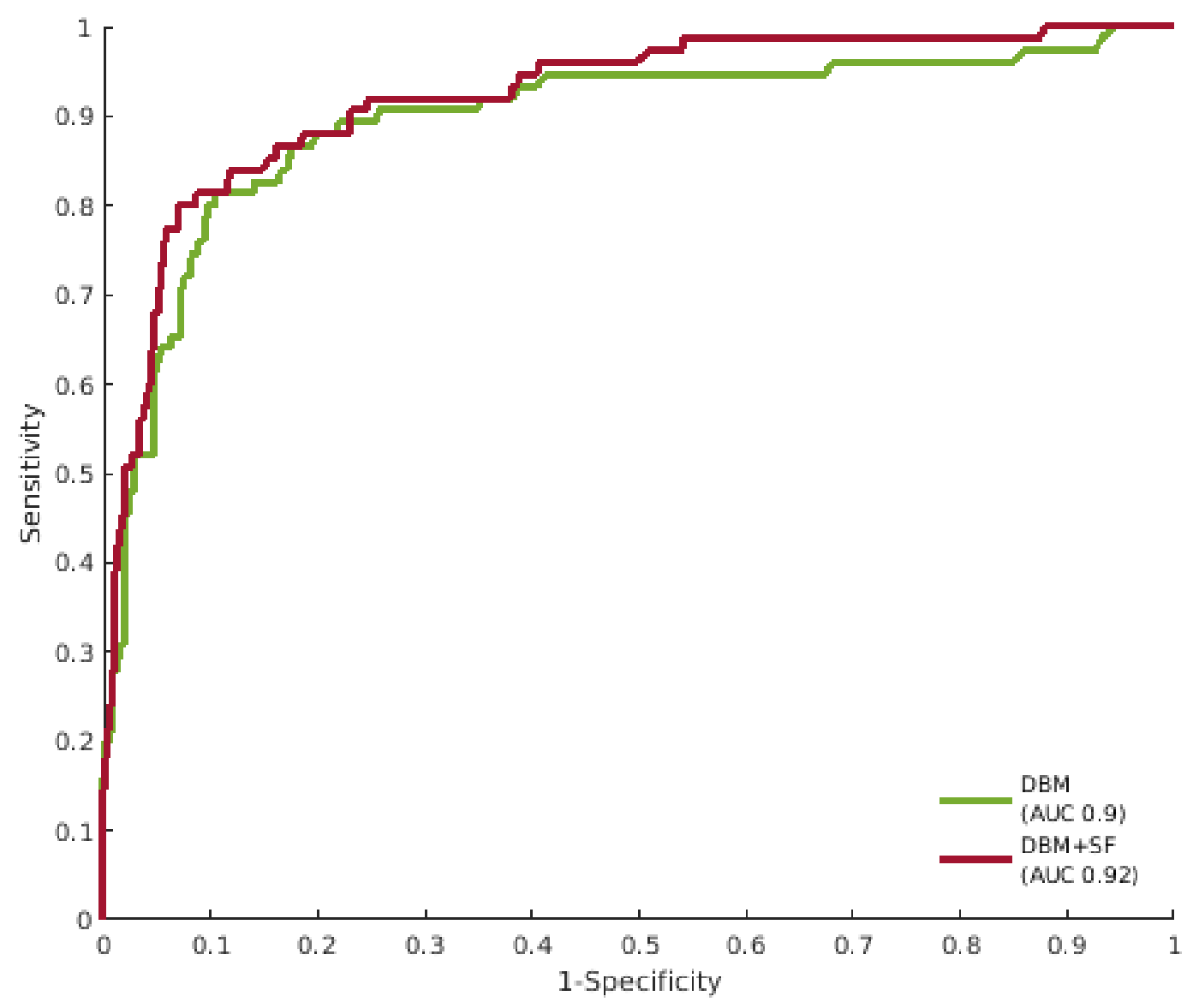

Figure 2. Receiver operating characteristic curves (ROC) for DBM and DBM+SF classifiers. Abbreviations: DBM: deformation-based morphometry; SF: semantic fluency; AUC: area under the curve. 

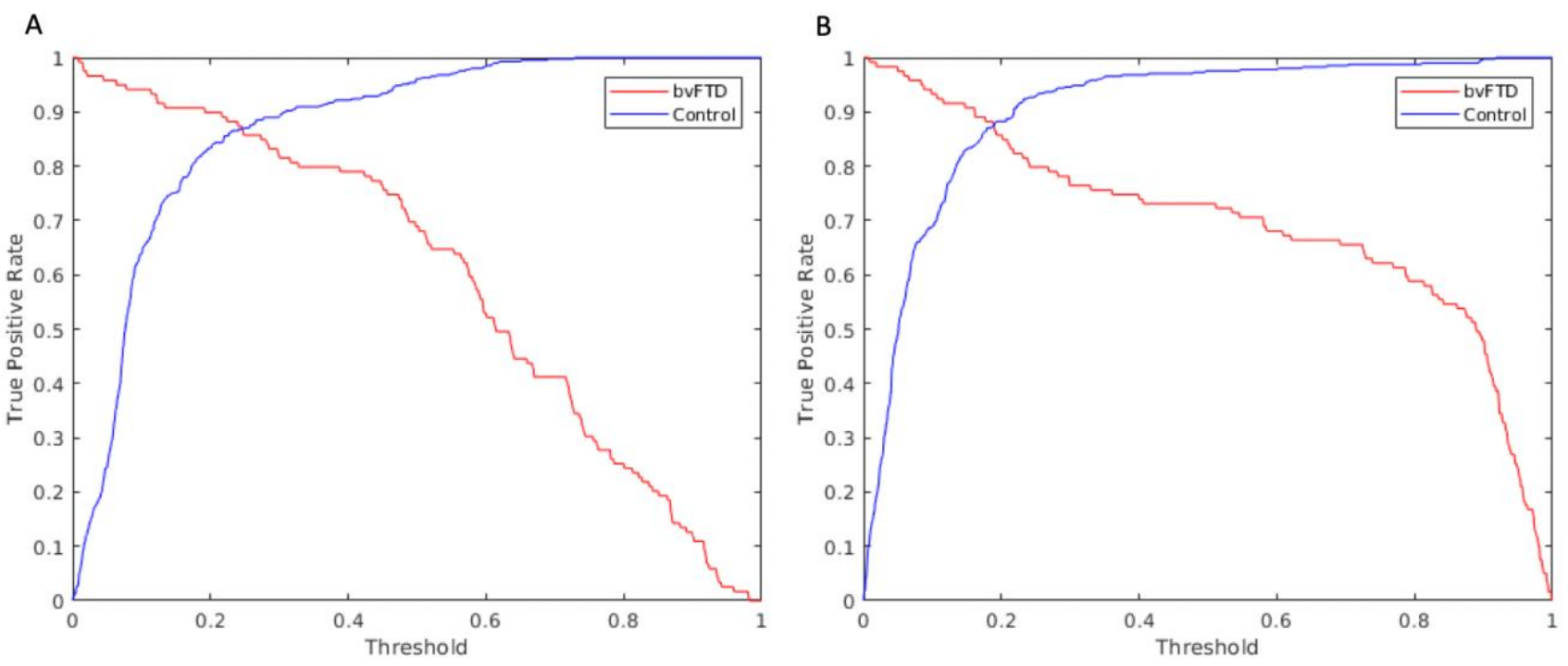

Figure 3. True positive rates for bvFTD and controls according to the probability score threshold for classification using DBM (panel A) or DBM + SF (panel B).

Abbreviations: bvFTD: behavioural-variant frontotemporal dementia. CNCs: cognitively normal controls. 
Table 2. Classification performance using DBM and DBM + SF

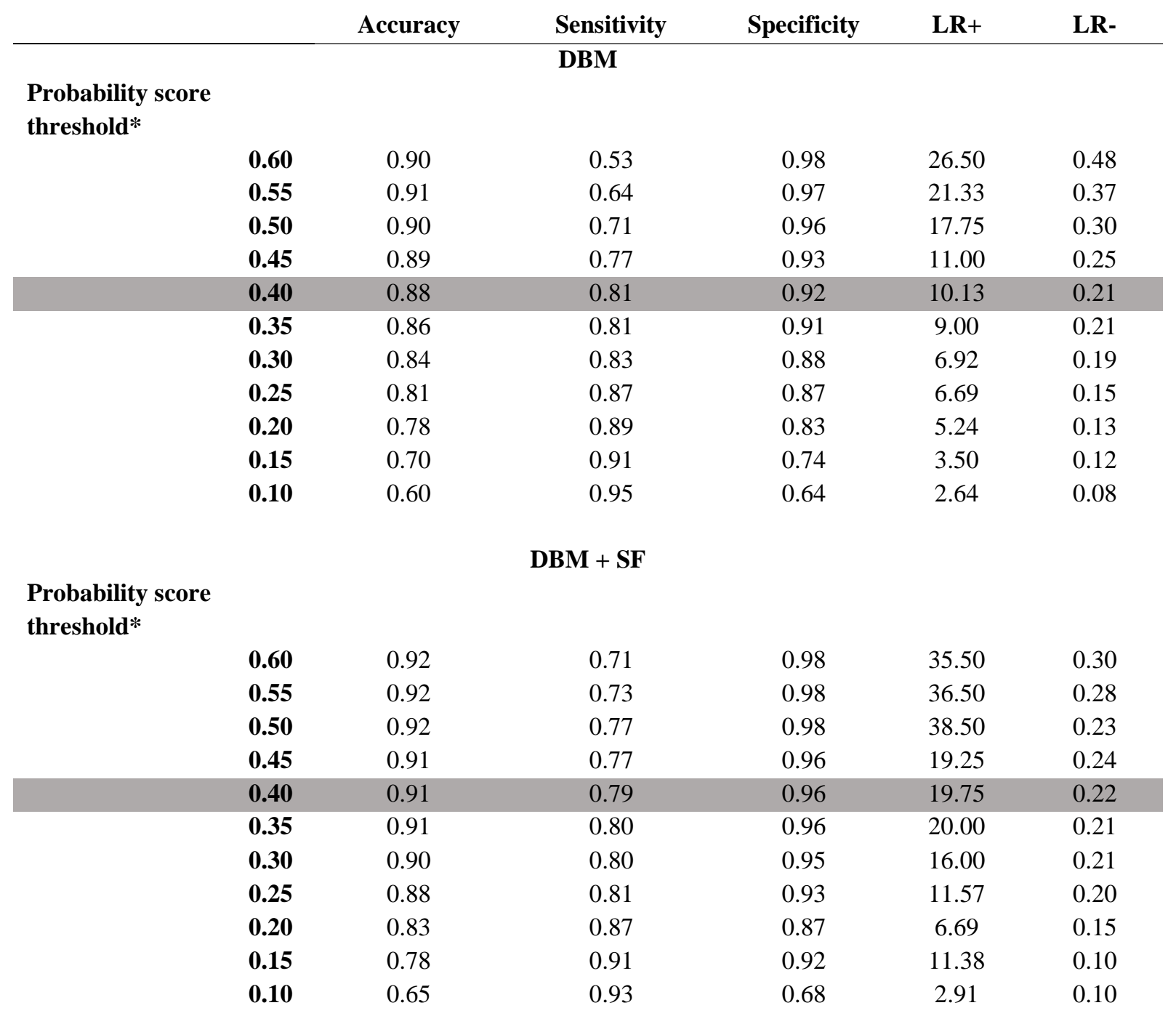

*Performances for each probability score threshold above which a subject is identified as bvFTD. Shaded rows correspond to the optimal cut-point estimated by Youden index.

Abbreviations: DBM: deformation-based morphometry; SF: semantic fluency score; LR+: positive likelihood ratio; LR-: negative likelihood ratio. 


\section{False negative cases within the validation cohort (GENFI2)}

The classification using DBM resulted in $19 \%$ of false negatives. These subjects were significantly younger than the bvFTD subjects correctly classified $(57 \pm 10$ vs. $66 \pm 7$ years respectively, $\mathrm{p}<$ $0.001)$ and the estimated time from onset was also shorter $(2 \pm 7$ years vs $6 \pm 5$ years; $\mathrm{p}=0.01)$. However, the disease duration was not significantly shorter than true positives (3.9[2.2-7.1] vs 5.3[3.6 8.2] years, respectively; $\mathrm{p}=0.16$ ). No significant differences were found in FTLD-CDR score between true positives and false negatives $(\mathrm{p}=0.07)$. The distribution of the genetic mutations did not show significant differences either between the false negatives and true positives. GRN corresponded to $22.7 \%$ of all false negatives and $25.4 \%$ of all true positives $(\mathrm{p}=$ 0.7); for $C 9$ orf72 the distribution was $45.5 \%$ and $54.7 \%$ respectively $(\mathrm{p}=0.5)$ while for $M A P T$ it was $31.8 \%$ of the false negatives and $18.9 \%$ of the correctly classified bvFTD ( $p=0.3)$

\section{False positive cases within the validation cohort}

Only 10 out of 247 CNCs (4\%) were erroneously classified as bvFTD. These subjects were significantly older than the subjects accurately classified as healthy subjects $(70 \pm 12$ years vs. $47 \pm 13$ years, respectively; $\mathrm{P}$ value $<0.001)$. No significant differences were found in the mean FTLD-CDR score $(\mathrm{P}$ value $=0.9)$. Of note, subjects with false positives had slightly lower mean MMSE scores $(28.27 \pm 2.2)$ compared to true negatives $(29.4 \pm 1$; P value $<0.001)$.

\section{Defining strategic cut-points}

Three cut-offs for both DBM and DBM+SF were defined by giving consideration to the sensitivity, specificity, positive and negative likelihood ratios of different points of the ROC: 1) the optimal cut-point according to Youden index; 2) a sensitive (i.e., "rule-out") cut-point; and 3) a specific 
(i.e., "rule-in") cut-point (Figure 4). The sensitivity, specificity, LR- and LR+ expressed in the Figure 4 were estimated for each of these defined cut-points (e.g., for probability score $=0.4)$.

Proposed thresholds for clinical decision-making for each classifier according to their likelihood ratios are proposed in Figure 4 (lower panels). A LR- <0.1 allows to reliably exclude (i.e., ruleout) bvFTD when the probability score is below 0.2 and below 0.1 for DBM and DBM+SF, respectively. Probability scores over 0.4 for DBM and over 0.25 for DBM+SF enable confident diagnosis (i.e., rule-in) of bvFTD with a $\mathrm{LR}+>10$. Corresponding likelihood ratios for different thresholds are shown in Table 2. 

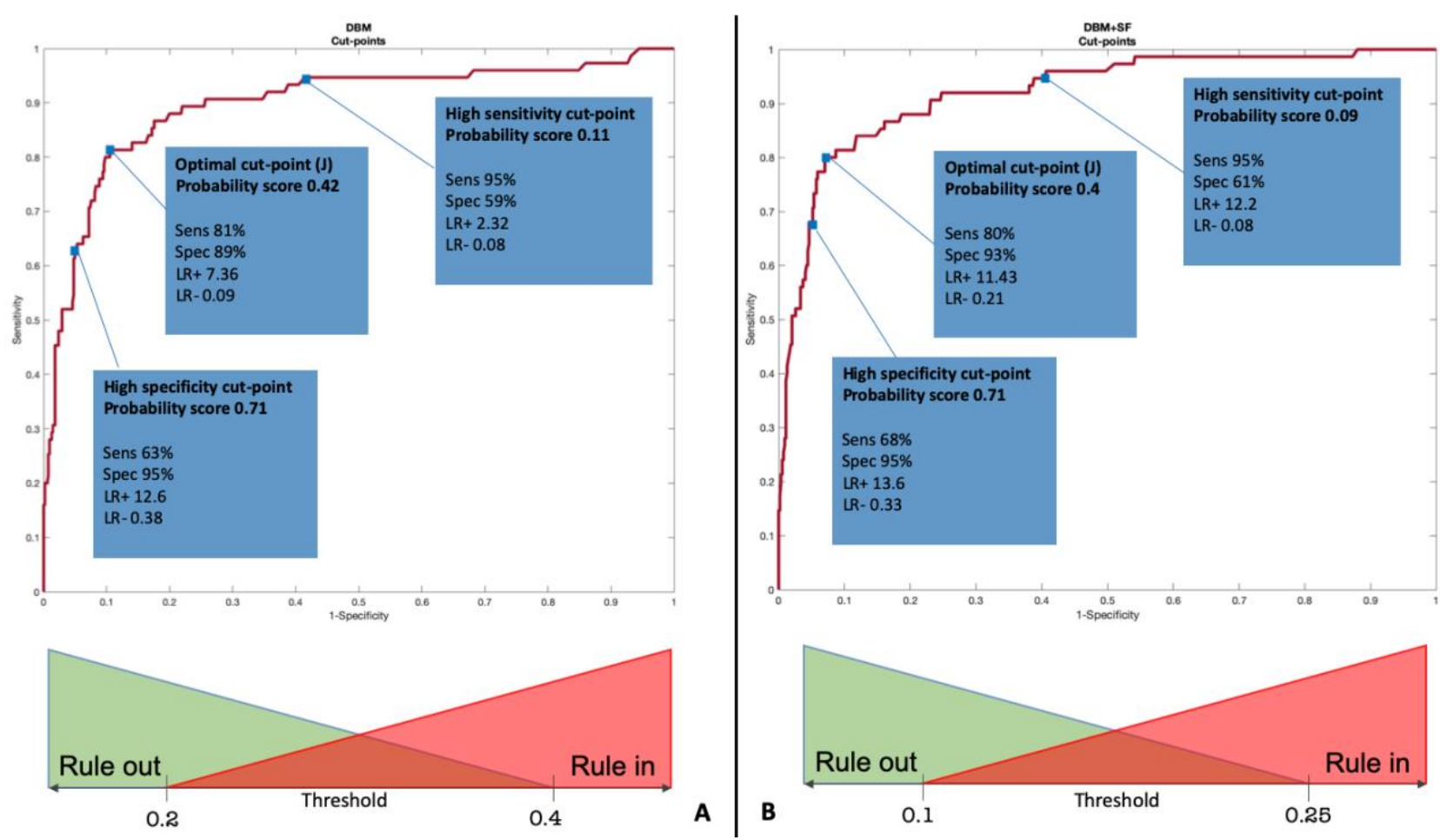

Figure 4. Strategic ROC cut points. 4A, upper panel: Cut points for DBM classifier. 4A, lower panel: Thresholds for diagnosis using DBM classifier in relation to LR+ and LR-. 4B, upper panel: Cut points for DBM+SF classifier. 4B, lower panel: Thresholds for diagnosis using $\mathrm{DBM}+\mathrm{SF}$ classifier in relation to LR+ and LR-.

Abbreviations: DBM: deformation-based morphometry; SF: semantic fluency; J: Youden index; Sens: sensitivity; Spec: specificity; LR+: positive likelihood ratio; LR-: negative likelihood ratio.

\section{DISCUSSION}

In the present study we built a random forest classifier using morphometric T1w MRI features for the individual prediction of bvFTD. The main findings are: 1) our random forest algorithm yielded areas under the curve of 0.90 and 0.92 using DBM and DBM+SF, respectively, in the independent validation cohort of genetically confirmed bvFTD cases; 2) the inclusion of a simple cognitive score (SF) improved the accuracies and specificity regardless of the probability threshold chosen, 
while reducing the false negative rate for probability scores $>0.5 ; 3$ ) we provide three cut-off values (a "statistically optimal" cut-point, a sensitive ("rule-out") cut-point and a specific ("rulein") cut-point) for both DBM and DBM+SF classifiers; and 4) our results show good positive and negative likelihood ratios proving its reliability in ruling in and out the disease.

The likelihood ratio is the percentage of patients with a given test result divided by the percentage of controls with the same results. Meaning that ill people should be more likely to have an abnormal result of a given test than healthy individuals. ${ }^{4041}$ For the DBM-only classifier in the independent GENFI2 validation cohort, the optimal threshold yielded an area under the curve (AUC) of 0.9 with $81 \%$ sensitivity and $92 \%$ specificity leading to a positive LR+ of 10.13 and negative LR- of 0.21 . Whereas, the AUC, sensitivity and specificity using the DBM+SF model were $0.92,79 \%$ and $96 \%$, respectively. These values result in LR+ of 19.75 and LR- of 0.22 . To keep in mind, a test is moderately good at ruling in disease when LR+ is greater than 2 and very good at doing it when LR+ is greater than $10 .{ }^{42}$ Furthermore, a test is moderately good at ruling out the disease with LR- below 0.5 and very good below 0.1 . Hence, using the optimal thresholds, both models are very good at excluding non bvFTD subjects and moderately good at confirming the disease.

Our results show that the proposed random forest classifier can accurately predict bvFTD in individual subjects in a completely independent validation cohort coming from a separate database. Furthermore, the GENFI2 validation cohort includes bvFTD patients with a definite diagnosis (positive genetic mutation). Of note, our algorithm was able to accurately classify patients with genetic bvFTD even though they tend to have more atypical atrophy patterns. ${ }^{21}$ The performance 
of our classifier is superior than the performance reported in several articles that have analyzed the standard diagnostic methods currently used in the clinical practice. Within a pathology-confirmed cohort, the sensitivity reported for the revised diagnostic criteria for bvFTD was $86 \%$ for possible diagnosis and $75 \%$ for probable bvFTD (with neuroimaging support). ${ }^{7}$ However, these criteria reported a sensitivity of $85 \%$ and specificity of $27 \%$ for possible bvFTD diagnosis in a clinically relevant cohort of patients with mixed behavioral changes, reaching $82 \%$ specificity when adding a compatible MRI scan. ${ }^{43}$ Within a cohort with late onset behavioral disorders, $70 \%$ sensitivity and 93\% specificity have been reported for structural MRI alone for bvFTD assessed by an experienced neuroradiologist. ${ }^{8}$ The latter results have comparable positive and negative likelihood ratios to ours, even though our method does not rely on the expertise of the radiological observer.

Previous studies classified bvFTD from a control group. ${ }^{9-13}$ The best AUC was reported by Raamana et al ${ }^{13}$ (AUC 0.938, 100\% sensitivity and $88 \%$ specificity). However, an important limitation is that they trained and tested on the same cohort which often results in an overestimate of the real rates when generalized to other populations. We addressed this particular issue using by using an independent dataset for validation. In addition, bvFTD diagnosis from the testing cohort was based on clinical criteria. Contrarily, the bvFTD subjects from our validation cohort have definite bvFTD diagnosis.

The performance of the classifier was tested on a held-out database which included multi-center and multi-scanner data from different scanner models of both $1.5 \mathrm{~T}$ and $3 \mathrm{~T}$ field strengths. This further reinforces the generalizability (i.e., external validity) of our results and ensures their applicability in a clinical scenario with different scanners, even with different magnetic field 
strengths. This certainly constitutes one of the two main strengths of this study. The second strength is that our performance was estimated using one of the gold standards for FTD diagnosis supported by the presence of a pathogenic mutation. Remarkably, our algorithm is based on standard structural T1w MRI and a simple cognitive test routinely acquired in the clinic, making for strong translational potential. On the other hand, the main limitation is that these results are yet to be validated prospectively in a clinically representative cohort including patients with diverse primary psychiatric disorders (a common differential diagnosis from bvFTD). ${ }^{44}$ The classification accuracy also remains to be demonstrated in cohorts with other types of dementias and cardiovascular comorbidities, as these were uncommon in our dataset and could have influenced our very high specificity. Finally, in our results the false negatives/positives were significantly younger/older than the subjects that were correctly classified. This is likely due to the fact that the age range for the validation dataset (GENFI: minimum age: 39y, maximum age:79y) was larger than the training set (FTLDNI, minimum age 46y, maximum age 75y). Subjects that were outside the operating range of the classifier were therefore more likely to be misclassified. Adding subjects with similar ages to the training dataset will likely improve the results. In addition, specifically for the false negative cases, although the difference did not reach statistical significance $(p=0.07)$, the false negatives had lower FTLD-CDR scores than the true positive cases, implying an earlier stage of the disease. It is plausible that such subjects with milder symptoms were not as well represented in FTLDNI given the difficulty of diagnosing bvFTD in the very mild stages when there is no known genetic mutation.

To conclude, we propose an automatic method using structural MRI alone (already available and routinely used in the clinic) and including a simple cognitive test that could be administered by 
any physician in few minutes for reliable individual prediction of bvFTD at the individual subject level. The main contributions of the method are its high accuracy along with its generalizability due to the use of a validation cohort coming from a different and independent database with multicenter and multi-scanner data. In addition, using a cohort with diagnosis of definite bvFTD adds reliability to the results. If validated in a prospective study, this algorithm has the potential to improve diagnostic accuracy, particularly in setting without access to specialized FTD care. 


\section{Acknowledgements}

Data collection and sharing for this project was funded by the Frontotemporal Lobar Degeneration Neuroimaging Initiative (National Institutes of Health Grant R01 AG032306). The study is coordinated through the University of California, San Francisco, Memory and Aging Center. FTLDNI data are disseminated by the Laboratory for Neuro Imaging at the University of Southern California.

Brain scan acquisition at the McConnell Brain Imaging was supported by the Brain Canada Foundation with support from Health Canada and the Canada Foundation for Innovation (CFI Project 34874).

This work was supported by Italian Ministry of Health (CoEN015 and Ricerca Corrente).

\section{Funding}

Dr. Collins receives funding from the Canadian Institutes of Health Research (MOP- 111169), les Fonds de Research Santé Quebec Pfizer Innovation fund, and an NSERC CREATE grant (4140438 - 2012). We would like to acknowledge funding from the Famille Louise \& André Charron.

Dr. Ducharme receives salary funding from the Fonds de Recherche du Québec - Santé.

\section{Competing interests}

Dr. Manera reports no disclosures

Dr. Dadar reports no disclosures

Dr. Collins is co-founder of True Positive Medical Devices.

Dr. Ducharme is the co-founder of Arctic Fox AI.

Dr. Ghidoni reports no disclosures 


\section{Appendix:}

\section{List of other GENFI consortium members:}

Sónia Afonso - Instituto Ciencias Nucleares Aplicadas a Saude, Universidade de Coimbra, Coimbra, Portugal Maria Rosario Almeida - Centre of Neurosciences and Cell Biology, Universidade de Coimbra, Coimbra, Portugal Sarah Anderl-Straub - Department of Neurology, Ulm University, Ulm, Germany Christin Andersson - Department of Clinical Neuroscience, Karolinska Institutet, Stockholm, Sweden Anna Antonell - Alzheimer's disease and other cognitive disorders unit, Neurology Department, Hospital Clinic, Institut d'Investigacions Biomèdiques, Barcelona, Spain

Silvana Archetti - Biotechnology Laboratory, Department of Diagnostics, ASST Brescia Hospital, Brescia, Italy Andrea Arighi - Fondazione IRCSS Ca’ Granda, Ospedale Maggiore Policlinico, Neurodegenerative Diseases Unit, Milan, Italy

Mircea Balasa - Alzheimer's disease and other cognitive disorders unit, Neurology Department, Hospital Clinic, Institut d'Investigacions Biomèdiques, Barcelona, Spain

Myriam Barandiaran - Neuroscience Area, Biodonostia Health Research Institute, Paseo Dr Begiristain sn, CP 20014, San Sebastian, Gipuzkoa, Spain

Nuria Bargalló - Radiology Department, Image Diagnosis Center, Hospital Clínic and Magnetic Resonance Image core facility, IDIBAPS, Barcelona, Spain

Robart Bartha - Department of Medical Biophysics, Robarts Research Institute, University of Western Ontario, London, Ontario, Canada

Benjamin Bender - Department of Diagnostic and Interventional Neuroradiology, University of Tuebingen, Tuebingen, Germany

Alberto Benussi - Centre for Neurodegenerative Disorders, Department of Clinical and Experimental Sciences, University of Brescia, Italy

Luisa Benussi - Istituto di Ricovero e Cura a Carattere Scientifico Istituto Centro San Giovanni di Dio Fatebenefratelli, Brescia, Italy

Valentina Bessi - Department of Neuroscience, Psychology, Drug Research, and Child Health, University of

Florence, Florence, Italy

Giuliano Binetti - Istituto di Ricovero e Cura a Carattere Scientifico Istituto Centro San Giovanni di Dio Fatebenefratelli, Brescia, Italy

Sandra Black - LC Campbell Cognitive Neurology Research Unit, Sunnybrook Research Institute, Toronto, Canada Martina Bocchetta - Dementia Research Centre, Department of Neurodegenerative Disease, UCL Institute of Neurology, Queen Square London, UK

Sergi Borrego-Ecija - Alzheimer's disease and other cognitive disorders unit, Neurology Department, Hospital Clinic, Institut d'Investigacions Biomèdiques, Barcelona, Spain

Jose Bras - Dementia Research Institute, Department of Neurodegenerative Disease, UCL Institute of Neurology, Queen Square, London, UK

Rose Bruffaerts - Laboratory for Cognitive Neurology, Department of Neurosciences, KU Leuven, Leuven, Belgium Paola Caroppo - Fondazione Istituto di Ricovero e Cura a Carattere Scientifico Istituto Neurologico Carlo Besta, Milan, Italy

David Cash - Dementia Research Centre, Department of Neurodegenerative Disease, UCL Institute of Neurology, Queen Square, London, UK

Miguel Castelo-Branco - Neurology Department, Centro Hospitalar e Universitário de Coimbra, Instituto de Ciências Nucleares Aplicadas à Saúde (ICNAS), Coimbra, Portugal

Rhian Convery - Dementia Research Centre, Department of Neurodegenerative Disease, UCL Institute of Neurology, Queen Square, London, UK

Thomas Cope - Department of Clinical Neuroscience, University of Cambridge, Cambridge, UK

Maura Cosseddu - Neurology, ASST Brescia Hospital, Brescia, Italy 
María de Arriba - Neuroscience Area, Biodonostia Health Research Institute, Paseo Dr Begiristain sn, CP 20014, San Sebastian, Gipuzkoa, Spain

Giuseppe Di Fede - Fondazione Istituto di Ricovero e Cura a Carattere Scientifico Istituto Neurologico Carlo Besta, Milan, Italy

Zigor Díaz - CITA Alzheimer, San Sebastian, Spain

Diana Duro - Faculty of Medicine, Universidade de Coimbra, Coimbra, Portugal

Chiara Fenoglio - University of Milan, Centro Dino Ferrari, Milan, Italy

Camilla Ferrari - Department of Neuroscience, Psychology, Drug Research, and Child Health, University of Florence, Florence, Italy

Carlos Ferreira - Instituto Ciências Nucleares Aplicadas à Saúde, Universidade de Coimbra, Coimbra, Portugal

Catarina B. Ferreira - Faculty of Medicine, University of Lisbon, Lisbon, Portugal

Toby Flanagan - Faculty of Biology, Medicine and Health, Division of Neuroscience and Experimental Psychology, University of Manchester, Manchester, UK

Nick Fox - Dementia Research Centre, Department of Neurodegenerative Disease, UCL Institute of Neurology, Queen Square, London, UK

Morris Freedman - Division of Neurology, Baycrest Centre for Geriatric Care, University of Toronto, Toronto, Canada Giorgio Fumagalli - Fondazione IRCSS Ca' Granda, Ospedale Maggiore Policlinico, Neurodegenerative Diseases Unit, Milan, Italy; Department of Neuroscience, Psychology, Drug Research and Child Health, University of Florence, Florence, Italy

Alazne Gabilondo - Neuroscience Area, Biodonostia Health Research Institute, Paseo Dr Begiristain sn, CP 20014, San Sebastian, Gipuzkoa, Spain

Roberto Gasparotti - Neuroradiology Unit, University of Brescia, Brescia, Italy

Serge Gauthier - Department of Neurology and Neurosurgery, McGill University, Montreal, Québec, Canada

Stefano Gazzina - Neurology, ASST Brescia Hospital, Brescia, Italy

Giorgio Giaccone - Fondazione Istituto di Ricovero e Cura a Carattere Scientifico Istituto Neurologico Carlo Besta, Milan, Italy

Ana Gorostidi - Neuroscience Area, Biodonostia Health Research Institute, Paseo Dr Begiristain sn, CP 20014, San Sebastian, Gipuzkoa, Spain

Caroline Greaves - Dementia Research Centre, Department of Neurodegenerative Disease, UCL Institute of Neurology, Queen Square London, UK

Rita Guerreiro - Dementia Research Institute, Department of Neurodegenerative Disease, UCL Institute of Neurology, London, UK

Carolin Heller - Dementia Research Centre, Department of Neurodegenerative Disease, UCL Institute of Neurology, Queen Square, London, UK

Tobias Hoegen - Department of Neurology, Ludwig-Maximilians-University of Munich, Munich, Germany

Begoña Indakoetxea - Cognitive Disorders Unit, Department of Neurology, Donostia University Hospital, Paseo Dr

Begiristain sn, CP 20014, San Sebastian, Gipuzkoa, Spain

Vesna Jelic - Division of Clinical Geriatrics, Karolinska Institutet, Stockholm, Sweden

Lize Jiskoot - Department of Neurology, Erasmus Medical Center, Rotterdam, The Netherlands

Hans-Otto Karnath - Section of Neuropsychology, Department of Cognitive Neurology, Center for Neurology \& Hertie-Institute for Clinical Brain Research, Tübingen, Germany

Ron Keren - University Health Network Memory Clinic, Toronto Western Hospital, Toronto, Canada

Maria João Leitão - Centre of Neurosciences and Cell Biology, Universidade de Coimbra, Coimbra, Portugal

Albert Lladó - Alzheimer's disease and other cognitive disorders unit, Neurology Department, Hospital Clinic, Institut d'Investigacions Biomèdiques, Barcelona, Spain

Gemma Lombardi - Department of Neuroscience, Psychology, Drug Research and Child Health, University of Florence, Florence, Italy

Sandra Loosli - Department of Neurology, Ludwig-Maximilians-University of Munich, Munich, Germany Carolina Maruta - Lisbon Faculty of Medicine, Language Research Laboratory, Lisbon, Portugal 
Simon Mead - MRC Prion Unit, Department of Neurodegenerative Disease, UCL Institute of Neurology, Queen Square, London, UK

Lieke Meeter - Department of Neurology, Erasmus Medical Center, Rotterdam, Netherlands

Gabriel Miltenberger - Faculty of Medicine, University of Lisbon, Lisbon, Portugal

Rick van Minkelen - Department of Clinical Genetics, Erasmus Medical Center, Rotterdam, The Netherlands

Sara Mitchell - LC Campbell Cognitive Neurology Research Unit, Sunnybrook Research Institute, Toronto, Canada

Katrina M Moore - Dementia Research Centre, Department of Neurodegenerative Disease, UCL Institute of Neurology, Queen Square, London, UK

Benedetta Nacmias - Department of Neuroscience, Psychology, Drug Research and Child Health, University of Florence, Florence, Italy

Mollie Neason - Dementia Research Centre, Department of Neurodegenerative Disease, UCL Institute of Neurology, Queen Square, London, UK

Jennifer Nicholas - Department of Medical Statistics, London School of Hygiene and Tropical Medicine, London, UK

Linn Öijerstedt - Department of Geriatric Medicine, Karolinska Institutet, Stockholm, Sweden

Jaume Olives - Alzheimer's disease and other cognitive disorders unit, Neurology Department, Hospital Clinic, Institut d'Investigacions Biomèdiques, Barcelona, Spain

Sebastien Ourselin - School of Biomedical Engineering \& Imaging Sciences, King's College London, London, UK

Alessandro Padovani - Centre for Neurodegenerative Disorders, Department of Clinical and Experimental Sciences, University of Brescia, Italy

Jessica Panman - Department of Neurology, Erasmus Medical Center, Rotterdam, The Netherlands

Janne Papma - Department of Neurology, Erasmus Medical Center, Rotterdam, The Netherlands

Georgia Peakman - Department of Neurodegenerative Disease, Dementia Research Centre, UCL Institute of Neurology, Queen Square, London, UK

Irene Piaceri - Department of Neuroscience, Psychology, Drug Research and Child Health, University of Florence,

Florence

Michela Pievani - Istituto di Ricovero e Cura a Carattere Scientifico Istituto Centro San Giovanni di Dio

Fatebenefratelli, Brescia, Italy

Yolande Pijnenburg - VUMC, Amsterdam, The Netherlands

Cristina Polito - Department of Biomedical, Experimental and Clinical Sciences "Mario Serio", Nuclear Medicine Unit, University of Florence, Florence, Italy

Enrico Premi - Stroke Unit, ASST Brescia Hospital, Brescia, Italy

Sara Prioni - Fondazione Istituto di Ricovero e Cura a Carattere Scientifico Istituto Neurologico Carlo Besta, Milan, Italy

Catharina Prix - Department of Neurology, Ludwig-Maximilians-University Munich, Germany

Rosa Rademakers - Department of Neurosciences, Mayo Clinic, Jacksonville, Florida, USA

Veronica Redaelli - Fondazione Istituto di Ricovero e Cura a Carattere Scientifico Istituto Neurologico Carlo Besta, Milan, Italy

Tim Rittman - Department of Clinical Neurosciences, University of Cambridge, Cambridge, UK

Ekaterina Rogaeva - Tanz Centre for Research in Neurodegenerative Diseases, University of Toronto, Toronto, Canada

Pedro Rosa-Neto - Translational Neuroimaging Laboratory, McGill University Montreal, Québec, Canada

Giacomina Rossi - Fondazione Istituto di Ricovero e Cura a Carattere Scientifico Istituto Neurologico Carlo Besta, Milan, Italy

Martin Rossor - Dementia Research Centre, Department of Neurodegenerative Disease, UCL Institute of Neurology, Queen Square, London, UK

Beatriz Santiago - Neurology Department, Centro Hospitalar e Universitário de Coimbra, Coimbra, Portugal Elio Scarpini - University of Milan, Centro Dino Ferrari, Milan, Italy; Fondazione IRCSS Ca' Granda, Ospedale Maggiore Policlinico, Neurodegenerative Diseases Unit, Milan, Italy 
Sonja Schönecker - Neurologische Klinik, Ludwig-Maximilians-Universität München, Munich, Germany

Elisa Semler - Department of Neurology, Ulm University, Ulm, Germany

Rachelle Shafei - Dementia Research Centre, Department of Neurodegenerative Disease, UCL Institute of Neurology, Queen Square, London, UK

Christen Shoesmith - Department of Clinical Neurological Sciences, University of Western Ontario, London, Ontario, Canada

Miguel Tábuas-Pereira - Centre of Neurosciences and Cell Biology, Universidade de Coimbra, Coimbra, Portugal

Mikel Tainta - Neuroscience Area, Biodonostia Health Research Institute, Paseo Dr Begiristain sn, CP 20014, San

Sebastian, Gipuzkoa, Spain

Ricardo Taipa - Neuropathology Unit and Department of Neurology, Centro Hospitalar do Porto - Hospital de Santo António, Oporto, Portugal

David Tang-Wai - University Health Network Memory Clinic, Toronto Western Hospital, Toronto, Canada

David L Thomas - Neuroradiological Academic Unit, UCL Institute of Neurology, London, UK

Hakan Thonberg - Center for Alzheimer Research, Division of Neurogeriatrics, Karolinska Institutet, Stockholm, Sweden

Carolyn Timberlake - Department of Clinical Neurosciences, University of Cambridge, Cambridge, UK

Pietro Tiraboschi - Fondazione Istituto di Ricovero e Cura a Carattere Scientifico Istituto Neurologico Carlo Besta, Milano, Italy

Emily Todd - Department of Neurodegenerative Disease, Dementia Research Centre, UCL Institute of Neurology, Queen Square, London, UK

Philip Vandamme - Neurology Service, University Hospitals Leuven, Belgium; Laboratory for Neurobiology, VIBKU Leuven Centre for Brain Research, Leuven, Belgium

Mathieu Vandenbulcke - Geriatric Psychiatry Service, University Hospitals Leuven, Belgium; Neuropsychiatry, Department of Neurosciences, KU Leuven, Leuven, Belgium

Michele Veldsman - Nuffield Department of Clinical Neurosciences, Medical Sciences Division, University of Oxford, UK

Ana Verdelho - Department of Neurosciences, Santa Maria Hospital, University of Lisbon, Portugal

Jorge Villanua - OSATEK Unidad de Donostia, San Sebastian, Gipuzkoa, Spain

Jason Warren - Dementia Research Centre, Department of Neurodegenerative Disease, UCL Institute of Neurology, Queen Square, London, UK

Carlo Wilke - Hertie Institute for Clinical Brain Research, University of Tuebingen, Tuebingen, Germany

Ione Woollacott - Dementia Research Centre, Department of Neurodegenerative Disease, UCL Institute of

Neurology, Queen Square, London, UK

Elisabeth Wlasich - Neurologische Klinik, Ludwig-Maximilians-Universität München, Munich, Germany

Henrik Zetterberg - Department of Neurodegenerative Disease, UCL Institute of Neurology, London, UK

Miren Zulaica - Neuroscience Area, Biodonostia Health Research Institute, Paseo Dr Begiristain sn, CP 20014, San

Sebastian, Gipuzkoa, Spain. 


\section{References:}

1. Seelaar H, Rohrer JD, Pijnenburg YA, et al. Clinical, genetic and pathological heterogeneity of frontotemporal dementia: a review. J Neurol Neurosurg Psychiatry 2011;82(5):476-86. doi: 10.1136/jnnp.2010.212225 [published Online First: 2010/10/26]

2. Onyike CU, Diehl-Schmid J. The epidemiology of frontotemporal dementia. Int Rev Psychiatry 2013;25(2):130-7. doi: 10.3109/09540261.2013.776523 [published Online First: 2013/04/25]

3. Rohrer JD, Guerreiro R, Vandrovcova J, et al. The heritability and genetics of frontotemporal lobar degeneration. Neurology 2009;73(18):1451-6. doi:

10.1212/WNL.0b013e3181bf997a [published Online First: 2009/11/04]

4. Cairns NJ, Bigio EH, Mackenzie IR, et al. Neuropathologic diagnostic and nosologic criteria for frontotemporal lobar degeneration: consensus of the Consortium for Frontotemporal Lobar Degeneration. Acta Neuropathol 2007;114(1):5-22. doi: 10.1007/s00401-0070237-2 [published Online First: 2007/06/21]

5. Bang J, Spina S, Miller BL. Frontotemporal dementia. Lancet 2015;386(10004):1672-82. doi: 10.1016/S0140-6736(15)00461-4 [published Online First: 2015/11/26]

6. Ducharme $S$, Dols $A$, Laforce $R$, et al. Recommendations to distinguish behavioural variant frontotemporal dementia from psychiatric disorders. Brain 2020 doi: 10.1093/brain/awaa018 [published Online First: 2020/03/05]

7. Rascovsky K, Hodges JR, Knopman D, et al. Sensitivity of revised diagnostic criteria for the behavioural variant of frontotemporal dementia. Brain 2011;134(Pt 9):2456-77. doi: 10.1093/brain/awr179 [published Online First: 2011/08/04]

8. Vijverberg EG, Wattjes MP, Dols A, et al. Diagnostic Accuracy of MRI and Additional [18F]FDG-PET for Behavioral Variant Frontotemporal Dementia in Patients with Late Onset Behavioral Changes. J Alzheimers Dis 2016;53(4):1287-97. doi: 10.3233/JAD160285 [published Online First: 2016/07/04]

9. Chow TW, Binns MA, Freedman M, et al. Overlap in frontotemporal atrophy between normal aging and patients with frontotemporal dementias. Alzheimer Dis Assoc Disord 2008;22(4):327-35. doi: 10.1097/WAD.0b013e31818026c4 [published Online First: 2008/08/13]

10. Mahoney CJ, Ridgway GR, Malone IB, et al. Profiles of white matter tract pathology in frontotemporal dementia. Hum Brain Mapp 2014;35(8):4163-79. doi: 10.1002/hbm.22468 [published Online First: 2014/02/11]

11. Meyer S, Mueller K, Stuke K, et al. Predicting behavioral variant frontotemporal dementia with pattern classification in multi-center structural MRI data. Neuroimage Clin 2017;14:656-62. doi: 10.1016/j.nicl.2017.02.001 [published Online First: 2017/03/30]

12. Moller C, Pijnenburg YA, van der Flier WM, et al. Alzheimer Disease and Behavioral Variant Frontotemporal Dementia: Automatic Classification Based on Cortical Atrophy for Single-Subject Diagnosis. Radiology 2016;279(3):838-48. doi: 10.1148/radiol.2015150220 [published Online First: 2015/12/15]

13. Raamana PR, Rosen $\mathrm{H}$, Miller B, et al. Three-Class Differential Diagnosis among Alzheimer Disease, Frontotemporal Dementia, and Controls. Front Neurol 2014;5:71. doi: 10.3389/fneur.2014.00071 [published Online First: 2014/05/27] 
14. Canu E, Agosta F, Mandic-Stojmenovic G, et al. Multiparametric MRI to distinguish early onset Alzheimer's disease and behavioural variant of frontotemporal dementia. Neuroimage Clin 2017;15:428-38. doi: 10.1016/j.nicl.2017.05.018 [published Online First: 2017/06/16]

15. Frings $L$, Yew $B$, Flanagan $E$, et al. Longitudinal grey and white matter changes in frontotemporal dementia and Alzheimer's disease. PLoS One 2014;9(3):e90814. doi: 10.1371/journal.pone.0090814 [published Online First: 2014/03/07]

16. Wang J, Redmond SJ, Bertoux M, et al. A Comparison of Magnetic Resonance Imaging and Neuropsychological Examination in the Diagnostic Distinction of Alzheimer's Disease and Behavioral Variant Frontotemporal Dementia. Front Aging Neurosci 2016;8:119. doi: 10.3389/fnagi.2016.00119 [published Online First: 2016/07/06]

17. Zhutovsky P, Vijverberg EGB, Bruin WB, et al. Individual Prediction of Behavioral Variant Frontotemporal Dementia Development Using Multivariate Pattern Analysis of Magnetic Resonance Imaging Data. J Alzheimers Dis 2019;68(3):1229-41. doi: 10.3233/JAD-181004 [published Online First: 2019/03/26]

18. McCarthy J, Collins DL, Ducharme S. Morphometric MRI as a diagnostic biomarker of frontotemporal dementia: A systematic review to determine clinical applicability. Neuroimage Clin 2018;20:685-96. doi: 10.1016/j.nicl.2018.08.028 [published Online First: 2018/09/16]

19. Whitwell JL, Boeve BF, Weigand SD, et al. Brain atrophy over time in genetic and sporadic frontotemporal dementia: a study of 198 serial magnetic resonance images. Eur J Neurol 2015;22(5):745-52. doi: 10.1111/ene.12675 [published Online First: 2015/02/17]

20. Whitwell JL, Weigand SD, Boeve BF, et al. Neuroimaging signatures of frontotemporal dementia genetics: C9ORF72, tau, progranulin and sporadics. Brain 2012;135(Pt 3):794806. doi: 10.1093/brain/aws001 [published Online First: 2012/03/01]

21. Cash DM, Bocchetta M, Thomas DL, et al. Patterns of gray matter atrophy in genetic frontotemporal dementia: results from the GENFI study. Neurobiol Aging 2018;62:19196. doi: 10.1016/j.neurobiolaging.2017.10.008 [published Online First: 2017/11/25]

22. Breiman L. Random Forests. Machine Learning 2001;45(1):5-32. doi: 10.1023/a:1010933404324

23. Rohrer JD, Nicholas JM, Cash DM, et al. Presymptomatic cognitive and neuroanatomical changes in genetic frontotemporal dementia in the Genetic Frontotemporal dementia Initiative (GENFI) study: a cross-sectional analysis. Lancet Neurol 2015;14(3):253-62. doi: 10.1016/S1474-4422(14)70324-2 [published Online First: 2015/02/11]

24. Folstein MF, Folstein SE, McHugh PR. "Mini-mental state". A practical method for grading the cognitive state of patients for the clinician. J Psychiatr Res 1975;12(3):189-98. doi: 10.1016/0022-3956(75)90026-6 [published Online First: 1975/11/01]

25. Nasreddine ZS, Phillips NA, Bedirian V, et al. The Montreal Cognitive Assessment, MoCA: a brief screening tool for mild cognitive impairment. J Am Geriatr Soc 2005;53(4):695-9. doi: 10.1111/j.1532-5415.2005.53221.x [published Online First: 2005/04/09]

26. Busner J, Targum SD. The clinical global impressions scale: applying a research tool in clinical practice. Psychiatry (Edgmont) 2007;4(7):28-37. [published Online First: 2007/07/01]

27. Borroni B, Agosti C, Premi E, et al. The FTLD-modified Clinical Dementia Rating scale is a reliable tool for defining disease severity in frontotemporal lobar degeneration: 
evidence from a brain SPECT study. Eur J Neurol 2010;17(5):703-7. doi: 10.1111/j.14681331.2009.02911.x [published Online First: 2010/01/07]

28. Mioshi E, Hsieh S, Savage $S$, et al. Clinical staging and disease progression in frontotemporal dementia. Neurology 2010;74(20):1591-7. doi: 10.1212/WNL.0b013e3181e04070 [published Online First: 2010/05/19]

29. Aubert-Broche B, Fonov VS, Garcia-Lorenzo D, et al. A new method for structural volume analysis of longitudinal brain MRI data and its application in studying the growth trajectories of anatomical brain structures in childhood. Neuroimage 2013;82:393-402. doi: 10.1016/j.neuroimage.2013.05.065 [published Online First: 2013/05/31]

30. Coupe $P$, Yger $P$, Prima $S$, et al. An optimized blockwise nonlocal means denoising filter for 3-D magnetic resonance images. IEEE Trans Med Imaging 2008;27(4):425-41. doi: 10.1109/TMI.2007.906087 [published Online First: 2008/04/09]

31. Sled JG, Zijdenbos AP, Evans AC. A nonparametric method for automatic correction of intensity nonuniformity in MRI data. IEEE Trans Med Imaging 1998;17(1):87-97. doi: 10.1109/42.668698 [published Online First: 1998/06/09]

32. Collins DL, Neelin P, Peters TM, et al. Automatic 3D intersubject registration of MR volumetric data in standardized Talairach space. J Comput Assist Tomogr 1994;18(2):192-205. [published Online First: 1994/03/01]

33. Avants BB, Epstein CL, Grossman M, et al. Symmetric diffeomorphic image registration with cross-correlation: evaluating automated labeling of elderly and neurodegenerative brain. Med Image Anal 2008;12(1):26-41. doi: 10.1016/j.media.2007.06.004 [published Online First: 2007/07/31]

34. Ashburner J, Friston KJ. Voxel-based morphometry--the methods. Neuroimage 2000;11(6 Pt 1):805-21. doi: 10.1006/nimg. 2000.0582 [published Online First: 2000/06/22]

35. Ashburner J, Hutton C, Frackowiak R, et al. Identifying global anatomical differences: deformation-based morphometry. Hum Brain Mapp 1998;6(5-6):348-57. [published Online First: 1998/10/27]

36. Manera AL, Dadar M, Collins DL, et al. Deformation based morphometry study of longitudinal MRI changes in behavioral variant frontotemporal dementia. Neuroimage: Clinical 2019;24 doi: https://doi.org/10.1016/j.nicl.2019.102079

37. Libon DJ, McMillan C, Gunawardena D, et al. Neurocognitive contributions to verbal fluency deficits in frontotemporal lobar degeneration. Neurology 2009;73(7):535-42. doi: 10.1212/WNL.0b013e3181b2a4f5 [published Online First: 2009/08/19]

38. Giovagnoli AR, Erbetta A, Reati F, et al. Differential neuropsychological patterns of frontal variant frontotemporal dementia and Alzheimer's disease in a study of diagnostic concordance. Neuropsychologia 2008;46(5):1495-504. doi: 10.1016/j.neuropsychologia.2007.12.023 [published Online First: 2008/02/12]

39. Hornberger $M$, Piguet $O$, Kipps $C$, et al. Executive function in progressive and nonprogressive behavioral variant frontotemporal dementia. Neurology 2008;71(19):1481-8. doi: 10.1212/01.wnl.0000334299.72023.c8 [published Online First: 2008/11/05]

40. Grimes DA, Schulz KF. Refining clinical diagnosis with likelihood ratios. Lancet 2005;365(9469):1500-5. doi: 10.1016/S0140-6736(05)66422-7 [published Online First: 2005/04/27] 
41. McGee S. Simplifying likelihood ratios. J Gen Intern Med 2002;17(8):646-9. doi: 10.1046/j.1525-1497.2002.10750.x [published Online First: 2002/09/06]

42. Doust J. Using probabilistic reasoning. BMJ 2009;339

43. Vijverberg EG, Dols A, Krudop WA, et al. Diagnostic Accuracy of the Frontotemporal Dementia Consensus Criteria in the Late-Onset Frontal Lobe Syndrome. Dement Geriatr Cogn Disord 2016;41(3-4):210-9. doi: 10.1159/000444849 [published Online First: 2016/05/11]

44. Ducharme S, Price BH, Larvie M, et al. Clinical Approach to the Differential Diagnosis Between Behavioral Variant Frontotemporal Dementia and Primary Psychiatric Disorders. Am J Psychiatry 2015;172(9):827-37. doi: 10.1176/appi.ajp.2015.14101248 [published Online First: 2015/09/02] 